\title{
What is the most promising treatment for Parkinson's disease: genes, cells, growth factors or none of the above?
}

“...it has to be realized that cell therapies are not a cure for Parkinson's disease..."

\section{KEYWORDS: cell replacement therapy $\approx$ dyskinesia - fetal dopaminergic neuron $\approx$ gene therapy $\approx$ induced pluripotent stem cell $\approx$ neurotrophic factor $\approx$ TRANSEURO}

Parkinson's disease (PD) is the second most common neurodegenerative disorder worldwide after Alzheimer's disease and has a broad spectrum of clinical manifestations, including motor and non-motor features with rest tremor, bradykinesia, rigidity and loss of postural reflexes being considered as the cardinal signs. It is pathologically defined by the severe and relatively selective loss of the dopaminergic neurons in the ventral tier of the substantia nigra pars compacta and the presence of $\alpha$-synuclein-positive Lewy bodies and Lewy neurites in specific regions of the nervous system. Braak and coworkers proposed a pathological staging scheme for PD after studying $\alpha$-synuclein staining in autopsied brains, hypothesizing that neural involvement spreads from the olfactory system and lower brainstem (and even possibly the enteric nervous system), and later involves more rostral brainstem structures and the cerebral cortex [1]. Thus, by the time patients present with motor symptoms of PD, there is extensive CNS pathology, $60 \%$ of the dopaminergic neurons in the substantia nigra have been lost and the level of dopamine in the striatum is $80 \%$ less than normal. Indeed, many now regard the disorder as starting at the synapse with secondary loss of the nigral dopaminergic neurons [2].

The principal aim of existing PD treatments is to restore the lowered dorsal striatal dopamine levels. However, dopamine-enhancing pharmacotherapies are limited by their efficacy and side-effects. They work most effectively in early disease when the nigrostriatal pathway is more intact because with disease progression the uptake and efficacy of the dopaminergic therapies becomes more erratic. Levodopa is currently the most widely used and effective oral medication for PD; however, many patients develop levodopa-induced dyskinesias and motor fluctuations 5-15 years after initiation of the drug. These complications occur in approximately $50 \%$ of levodopa-treated patients who have received levodopa for more than 5 years, in $80 \%$ of patients treated for 10 years, and in nearly all patients with young-onset disease. In a bid to control these motor fluctuations, patients' therapy may need to be escalated to include more intensive pharmacotherapies such as infusions of subcutaneous apomorphine and jejunal Duodopa ${ }^{\circledR}$ infusions. In addition, deep brain stimulation (DBS) surgery, either of the subthalamic nucleus or the globus pallidus pars interna, can be useful for treating PD patients at this stage when the motor fluctuations and dyskinesias cannot be adequately managed with pharmacological manipulation. The 5-year follow-up of patients who have received bilateral stimulation of the subthalamic nucleus in PD clearly shows that their improvement in motor function is sustained over time. However, disability still progresses from year to year, indicating ongoing degeneration in both dopaminergic and nondopaminergic sites. Furthermore, DBS is also associated with significant cognitive and psychiatric side effects, including severe depression, impulsivity and apathy, all of which may help explain the discrepancy between changes in quality-of-life assessment and motor disability in patients having had this therapy [3].

None of the currently available therapies for PD can slow or halt the disease progression. Therefore, there is a clear need for new therapies in PD that not only are capable of providing dopamine replacement more effectively, but also serve to replace or protect the substantia nigra pars compacta dopamine neurons from further degeneration. In a bid to meet this clinical challenge, novel therapeutic approaches have evolved over the last 20 years, which include a variety of gene therapy approaches, neurotrophic factor delivery and cell replacement therapies.

Three main strategies under investigation using gene transfer for targeted protein expression include:

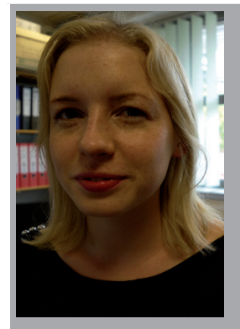

Gemma Cummins Cambridge Centre for Brain Repair \& the Department of Neurology, Department of Clinical Neurosciences, University of Cambridge, Cambridge, UK

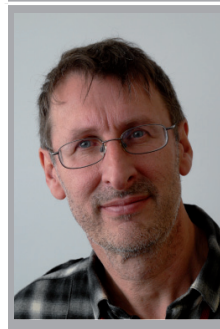

Roger A Barker Author for correspondence: Cambridge Centre for Brain Repair \& the Department of Neurology, Department of Clinical Neurosciences, University of Cambridge. Cambridge, UK Cambridge, UK
Tel.: +441223331160 Fax: +441223331174 rab46@cam.ac.uk 
- Improving the availability of therapeutic levels of dopamine to the striatum by delivering synthetic enzymes for its production;

- The induction of glutamic acid decarboxylase in the subthalamic nucleus;

- Protecting and restoring nigrostriatal neuronal function through local trophic factor delivery.

In this article we will only be discussing this latter approach that has involved using the glial cell line-derived neurotrophic factor (GDNF)-like growth factor, neurturin (NTN).

In the original open-label study using NTN, its primary safety end point was successfully met [4]. However, in a Phase II randomized control trial, involving 58 patients with advanced bilateral idiopathic PD, intraputaminal AAV2neurturin was not shown to be superior to sham surgery when assessed using the unified Parkinson's disease rating scale (UPDRS) motor 'off' score at 12 months, although positive results were seen in a subgroup of patients who were assessed after 18 months. The authors suggested this may be due to the dose of NTN used and a loss of retrograde transport of the growth factor to the substantia nigra as a result of dystrophic striatal projections. In addition, targeting advanced patients with striatal injections when there is almost no striatal dopaminergic fibers to rescue may also have accounted for the negative trial result. As a result a new study has been undertaken where rAAV2-NTN has been delivered into the substantia nigra, as well as the putamen in earlier stage patients who will then be followed up over longer time periods [5].

Neurotrophic factors are essential for neuronal survival, function and recovery, and GDNF in particular has a specific trophism for the nigral dopaminergic neurons [6]. In two small, open-label trials in PD patients, direct infusion of GDNF to the putamen led to significantly improved motor scores as assessed by the UPDRS, which were lost when the agent stopped being delivered $[7,8]$. However, when taken to a double-blind placebo-controlled study, it did not show the same level of efficacy [9]. The reason for this failure of the double-blind placebocontrolled trial to show the same results as the open-label studies has been the subject of much debate, especially given that in the original studies there was PET and post-mortem evidence of dopaminergic fiber sprouting around the site of the infusion. This debate has included how the GDNF was delivered and at what dose $[6,10]$. In addition, safety concerns were also raised, as anti-GDNF antibodies were found in a subset of treated patients, leading to concerns that an immune response might ensue after long-term treatment. Further review of earlier primate data also revealed cerebellar Purkinjie cell degeneration suggesting that the agent may be toxic, all of which led to a cessation of all GDNF trials, which in retrospect may have been premature [11]. Recently new trials using convectionenhanced delivery of GDNF are being planned in patients with PD [12].

\section{"...many now regard the disorder as starting at the synapse..."}

Clinical transplantation trials as a treatment for PD were started in the late 1980s after preclinical studies demonstrated that grafts of fetal dopaminergic neurons can survive for long periods, reinnervate the host striatum and secrete dopamine in a regulated fashion in animal models of PD. These initial open-label studies showed great promise and continue to do so, with some patients a quarter of a century into their illness, and up to 15 years after grafting, being off all dopaminergic treatment with UPDRS motor scores similar or better than that which they had when diagnosed. However, even within these open-label studies there were variable outcomes between patients in terms of motor recovery [13-17]. Nevertheless, encouraged by these open-label studies, the NIH funded two double-blind placebo-controlled trials of fetal ventral mesencephalon (VM) transplantation in patients with advanced PD and found in both cases no benefit using the primary trial end point $[18,19]$. The reason for this has again been debated and while it is not clear why these disparate results were seen, it may relate to issues of patient selection, tissue preparation and implantation, as well as the type and duration of immunotherapy adopted along with trial design and primary end points chosen. Taking all of these studies as a whole (open-label and double-blind trials), it appears that transplants work most effectively in younger patients with earlier stages of disease and with dopaminergic loss confined principally to the dorsal striatum [20,21].

In addition, these NIH-sponsored studies were the first to report that significant number of patients transplanted with foetal VM cells developed graft-induced dyskinesias (GIDs), in some cases these were so severe that they subsequently required DBS surgery as a corrective measure; surgery that was very effective. The reason for 
these GIDs is unknown, but two major theories have arisen to explain them. The first is that the transplanted tissue was not delivered evenly across the striatal complex, creating dopaminergic hot spots [22]. The other relates to the contaminating serotoninergic neurons within the VM graft. Human fetal VM tissue contains both dopaminergic and serotonergic neuroblasts and it has been proposed that mishandling of dopamine by the serotonin neurons could drive the GIDs if there are insufficient dopaminergic neurons within the graft to counter this [23]. This latter theory has received support in that two patients with GIDs were relieved of these abnormal movements when given buspirone acutely [24], and so in the future GIDs could be circumvented if a more enriched dopaminergic cell source could be used, as may be possible with stem cells (see below).

"...new trials using convection-enhanced delivery of glial cell line-derived neurotrophic factor are being planned in patients with Parkinson's disease."

Whilst neuropathological studies have demonstrated proof-of-graft survival and striatal reinnervation up to 16 years after transplantation of fetal VM tissue in patients with PD, they have also revealed that a small proportion of the graft- derived dopaminergic neurons contain Lewy bodies $[25,26]$. Given that these grafted dopamine neurons are at most 15 years old, it suggests that the disease may have spread from the host PD brain to the grafted cells, which has obvious implications for future cell therapies. However, the number of cells having such pathology was small and it is clear that some patients have extremely well-functioning grafts years after the cells were transplanted. Thus, whilst of interest, not least in terms of the possibility that PD is a prion-like disease [27], its significance for the future of cell-based therapies in PD is not great.

While these human foetal VM (hfVM) tissue transplant trials have produced a mixture of outcomes, they have nevertheless provided proof-ofprinciple evidence that this approach can restore striatal dopaminergic function and provide significant functional benefit in some patients with PD. As a result, new trials using hfVM cells are being planned, such as the TRANSEURO project that will seek to take advantage of the lessons learned from these earlier studies. In particular, this new trial will use carefully selected younger patients with early-stage PD and will optimize tissue preparation to reduce the risk of GIDs. This trial will also provide useful insights into the optimal way of designing protocols for future stem cell-based clinical trials.

HfVM tissue transplantation is thought unlikely ever to become a routine treatment for PD given the logistical and ethical problems associated with harvesting hfVM tissue. Stem cell therapies on the other hand are an appealing alternative given the relative ease with which they can be generated, the purity of the cells obtained and their potential to repair degenerating or injured dopaminergic neural circuits in animal models of disease. In particular, human embryonic stem cell (ESC)-derived dopaminergic neuroblasts have recently been shown to survive and have functional benefits in a variety of animal models of PD [28]. However, the clinical translation of such cells has been hampered by safety concerns, as well as issues to do with whether the dopaminergic neurons so generated are truly nigral dopaminergic neurons. Recently, these two concerns have been addressed using a novel approach by Studer et al. for differentiating human ESCs to dopaminergic nigral neurons, which has now set the scene for such cells entering the clinical arena in the relatively near future [29].

Human ESCs, while holding many attractions, bring with them many ethical problems, and thus the search for more ethically neutral cell sources has been undertaken. Induced pluripotent stem (iPS) cells have the potential to negate the need to use contentious embryonic tissue, as well as potentially remove the requirement for post-transplantation immunosuppressive therapies. iPS cell-derived dopaminergic neurons have displayed encouraging therapeutic effects in rodent PD models [30]. However, these cells are not without risks, including issues of tumorigenesis and rejection [31], as well as concerns regarding whether these autologous grafted cells have a genetic background that would ultimately mean they will succumb to the disease process post-grafting. In this respect, Sanchez-Danes et al. discovered that iPS derived dopaminergic neurons derived from patients with idiopathic PD have subtle abnormalities within them [32]. Thus, iPS cells in PD may prove more useful for the in vitro cell modelling of disease that could lead to greater understanding of its pathogenesis.

Recently, it has become possible to directly reprogram fibroblasts into functional induced neurons, including nigral dopaminergic neurons [33]. This conversion of one somatic cell form into another is an exciting new discovery in the 
field of stem cell medicine, as cells do not pass through a pluripotent state, making the tumorgenicity risk associated with other forms of stem cells sources less of an issue. However, it remains to be seen whether these grafted cells will be able to induce sustained functional effects in animal models of PD, before attempting clinical translation to patients.

Finally, it has to be realized that cell therapies are not a cure for PD as they are not able to treat many of the non-motor features associated with the disease, which are caused by degeneration of nondopaminergic neuronal systems. In order for cell-based therapies to be successful and clinically competitive, they will have to show sustainable improvement in the relevant motor features of PD and quality of life while demonstrating a good safety profile.

In summary there are many exciting new reparative strategies being pursued in the treatment of PD. All have run into problems, in part by the premature move to double-blind placebo-controlled trials ahead of the optimization of the therapy being used. Nevertheless, these trials have been highly informative, and through lessons learnt from them, new trials are now being planned which have the potential not to cure PD, but to significantly change the course of the natural history of treated PD for the better.

\section{Financial \& competing interests disclosure}

This work was supported by a NIHR award of a biomedical research centre to Addenbrooke's Hospital and the University of Cambridge. G Cummins is supported by a Van Geest Foundation scholarship. The authors have no other relevant affiliations or financial involvement with any organization or entity with a financial interest in or financial conflict with the subject matter or materials discussed in the manuscript apart from those disclosed.

No writing assistance was utilized in the production of this manuscript.

\section{References}

1 Braak H, Tredici KD, Rüb U, de Vos RA, Jansen Steur EN, Braak E. Staging of brain pathology related to sporadic Parkinson's disease. Neurobiol. Aging 24(2), 197-211 (2003).

2 Day M, Wang Z, Ding J et al. Selective elimination of glutamatergic synapses on striatopallidal neurons in Parkinson disease models. Nat. Neurosci. 9(2), 251-259 (2006).

3 Strutt AM, Simpson R, Jankovic J, York MK. Changes in cognitive-emotional and physiological symptoms of depression following STN-DBS for the treatment of Parkinson's disease. Eur. J. Neurol. 19(1), 121-127 (2012).

4 Marks WJ Jr, Ostrem JL, Verhagen L et al. Safety and tolerability of intraputaminal delivery of CERE-120 (adeno-associated virus serotype 2-neurturin) to patients with idiopathic Parkinson's disease: an open-label, Phase I trial. Lancet Neurol. 7(5), 400-408 (2008).

5 Marks WJ Jr, Bartus RT, Siffert J et al. Gene delivery of AAV2-neurturin for Parkinson's disease: a double-blind, randomised, controlled trial. Lancet Neurol. 9(12), 1164-1172 (2010).

6 Sherer TB, Fiske BK, Svendsen CN, Lang AE, Langston JW. Crossroads in GDNF therapy for Parkinson's disease. Mov. Disord. 21(2), 136-141 (2006).

7 Gill SS, Patel NK, Hotton GR et al. Direct brain infusion of glial cell line-derived neurotrophic factor in Parkinson disease. Nat. Med. 9(5), 589-595 (2003).
8 Slevin JT, Gerhardt GA, Smith CD, Gash DM, Kryscio R, Young B. Improvement of bilateral motor functions in patients with Parkinson disease through the unilateral intraputaminal infusion of glial cell line-derived neurotrophic factor. J. Neurosurg. 102(2), 216-222 (2005).

9 Lang AE, Gill S, Patel NK et al. Randomized controlled trial of intraputamenal glial cell line-derived neurotrophic factor infusion in Parkinson disease. Ann. Neurol. 59(3), 459-466 (2006).

10 Patel NK, Gill SS. GDNF delivery for Parkinson's disease. Acta Neurochir. Suppl. 97(Pt 2), 135-154 (2007).

11 Barker RA. Parkinson's disease and growth factors - are they the answer? Parkinsonism Relat. Disord. 15(Suppl. 3), S181-S184 (2009).

12 Richardson RM, Kells AP, Rosenbluth KH et al. Interventional MRI-guided putaminal delivery of AAV2-GDNF for a planned clinical trial in Parkinson's disease. Mol. Ther. 19(6), 1048-1057 (2011).

13 Madrazo I, Leon V, Torres C et al. Transplantation of fetal substantia nigra and adrenal medulla to the caudate nucleus in two patients with Parkinson's disease. N. Engl. J. Med. 318(1), 51 (1988).

14 Lindvall O, Rehncrona S, Brundin P et al. Human fetal dopamine neurons grafted into the striatum in two patients with severe Parkinson's disease. A detailed account of methodology and a 6-month follow-up. Arch. Neurol. 46(6), 615-631 (1989).

15 Spencer DD, Robbins RJ, Naftolin F et al. Unilateral transplantation of human fetal mesencephalic tissue into the caudate nucleus of patients with Parkinson's disease. N. Engl. J. Med. 327(22), 1541-1548 (1992).

16 Peschanski M, Defer G, N'guyen JP et al. Bilateral motor improvement and alteration of L-dopa effect in two patients with Parkinson's disease following intrastriatal transplantation of foetal ventral mesencephalon. Brain 117(Pt 3), 487-499 (1994).

17 Hauser RA, Freeman TB, Snow BJ et al. Long-term evaluation of bilateral fetal nigral transplantation in Parkinson disease. Arch. Neurol. 56(2), 179-187 (1999).

18 Freed CR, Breeze RE, Rosenberg NL et al. Survival of implanted fetal dopamine cells and neurologic improvement 12 to 46 months after transplantation for Parkinson's disease. N. Engl. J. Med. 327(22), 1549-1555 (1992).

19 Olanow CW, Goetz CG, Kordower JH et al. A double-blind controlled trial of bilateral fetal nigral transplantation in Parkinson's disease. Ann. Neurol. 54(3), 403-414 (2003).

20 Lindvall O, Bjorklund A. Cell therapy in Parkinson's disease. NeuroRx 1(4), 382-393 (2004).

21 Dyson SC, Barker RA. Cell-based therapies for Parkinson's disease. Expert Rev. Neurother. 11(6), 831-844 (2011).

22 Ma Y, Feigin A, Dhawan V et al. Dyskinesia after fetal cell transplantation for parkinsonism: a PET study. Ann. Neurol. 52(5), 628-634 (2002).

23 Barker RA, Kuan WL. Graft-induced dyskinesias in Parkinson's disease: what is it all about? Cell Stem Cell 7(2), 148-149 (2010). 
24 Politis M, Wu K, Loane C et al. Serotonergic neurons mediate dyskinesia side effects in Parkinson's patients with neural transplants. Sci. Transl. Med. 2(38), 38ra46 (2010).

25 Kordower JH, Chu Y, Hauser RA, Freeman TB, Olanow CW. Lewy body-like pathology in long-term embryonic nigral transplants in Parkinson's disease. Nat. Med. 14(5), 504-506 (2008).

26 Li JY, Englund E, Holton JL et al. Lewy bodies in grafted neurons in subjects with Parkinson's disease suggest host-to-graft disease propagation. Nat. Med. 14(5), 501-503 (2008).

27 Olanow CW, Prusiner SB. Is Parkinson's disease a prion disorder? Proc. Natl Acad. Sci. USA 106(31), 12571-12572 (2009).
28 Kim JH, Auerbach JM, Rodriguez-Gomez JA et al. Dopamine neurons derived from embryonic stem cells function in an animal model of Parkinson's disease. Nature 418(6893), 50-56 (2002).

29 Kriks S, Shim JW, Piao J et al. Dopamine neurons derived from human ES cells efficiently engraft in animal models of Parkinson's disease. Nature 480 (7378), 547-551 (2011).

30 Hargus G, Cooper O, Deleidi M et al. Differentiated Parkinson patient-derived induced pluripotent stem cells grow in the adult rodent brain and reduce motor asymmetry in Parkinsonian rats. Proc. Natl Acad. Sci. USA 107(36), 15921-15926 (2010).
31 Brederlau A, Correia AS, Anisimov SV et al. Transplantation of human embryonic stem cell-derived cells to a rat model of Parkinson's disease: effect of in vitro differentiation on graft survival and teratoma formation. Stem Cells 24(6), 1433-1440 (2006).

32 Sanchez-Danes A, Richaud-Patin Y, Carballo-Carbajal I et al. Disease-specific phenotypes in dopamine neurons from human iPS-based models of genetic and sporadic Parkinson's disease. EMBO Mol. Med. 4(5), 380-395 (2012).

33 Vierbuchen T, Ostermeier A, Pang ZP, Kokubu Y, Sudhof TC, Wernig M. Direct conversion of fibroblasts to functional neurons by defined factors. Nature 463(7284), 1035-1041 (2010). 